\title{
Crystal Structure, SAR, DFT and Hirshfeld analysis of imidazo thiadiazoles
}

\author{
$\mathrm{G} \mathrm{N}$ Anil Kumar $^{1}, \mathrm{M} \mathrm{K}$ Kokila $^{2}$, Sujeet Kumar ${ }^{3}$, Subhas S Karki ${ }^{3}$ \\ ${ }^{1}$ Department Of Physics, Ramaiah Institute Of Technology, Bengaluru, India, ${ }^{2}$ Department of Physics, Bangalore University, \\ Bangalore, India, ${ }^{3}$ Department of Pharmaceutical Chemistry, KLE University College of Pharmacy, Bengaluru, India \\ E-mail: anilgn@msrit.edu
}

The design of new molecules possessing potential biological activity is important area of research. Thiadiazole is a versatile moiety that exhibits a wide variety of biological activities since it acts as a constrained pharmacophore in many drugs such as acetazolamide, methazolamide, sulfamethazole, etc., In this context, a series of 2,5,6 imidazo[2,1-b][1,3,4]thiadiazole derivatives with various structural features were designed. The derivatives were found to exhibit cytotoxic activity against human cervix carcinoma as well as murine leukemia cells. Further, combined crystal structure and the structure activity relationship studies (SAR) indicated that, formyl and thiocyanate substituents at C5 resulted good cytotoxic activity against several tumor cell lines, which was superior to standard anti-tumor drugs such as melphalan or Levamisole[1]. Substitution of bromine at C-5 position did not produce any improvement in cytotoxicity activity.

The crystal structure investigation of series of 2,5,6-substituted imidazo[2,1-b][1,3,4]thiadiazoles has been carried out to understand the influence of various substituents especially halogens on the conformation of imidazo thiadiazole moiety.[2] Single crystals obtained by slow evaporation using ethanol and DMF mixture. Single crystal diffraction experiments were performed at $293 \mathrm{~K}$ on an Oxford Diffraction Xcalibur diffractometer. All structures were solved by direct methods and refined using the SHELXS and SHELXL programs respectively. Geometry optimizations for all investigated compounds were performed using the B3LYP basis set starting from crystallographically determined structures. Hirshfeld surfaces were calculated and plotted using Crystal Explorer.

The conformational changes in crystal structures have been derived in these classes of compounds, bring out the subtle differences in intermolecular interactions with respect to different substitutions. In most of the compounds, classical intermolecular hydrogen bonds are not present where packing of molecules is governed by weak interactions involving halogens. It also appears that halogen interactions along with classical hydrogen bonds influence the generation of molecular motifs in crystalline lattices. Further, it clearly shows that halogen interactions provide an additional stability to the crystal structures. The detailed study of interactions in all the compounds and their structure activity relation studies will be presented.

[1] Sujeet Kumar et al., European Journal of Medicinal Chemistry (2014), 84, 687-697.

[2] A. Sowmya, G. N. Anil Kumar, Sujeet Kumar and Subhas S. Karki, Acta Cryst. (2016). E72, 1460-1462.

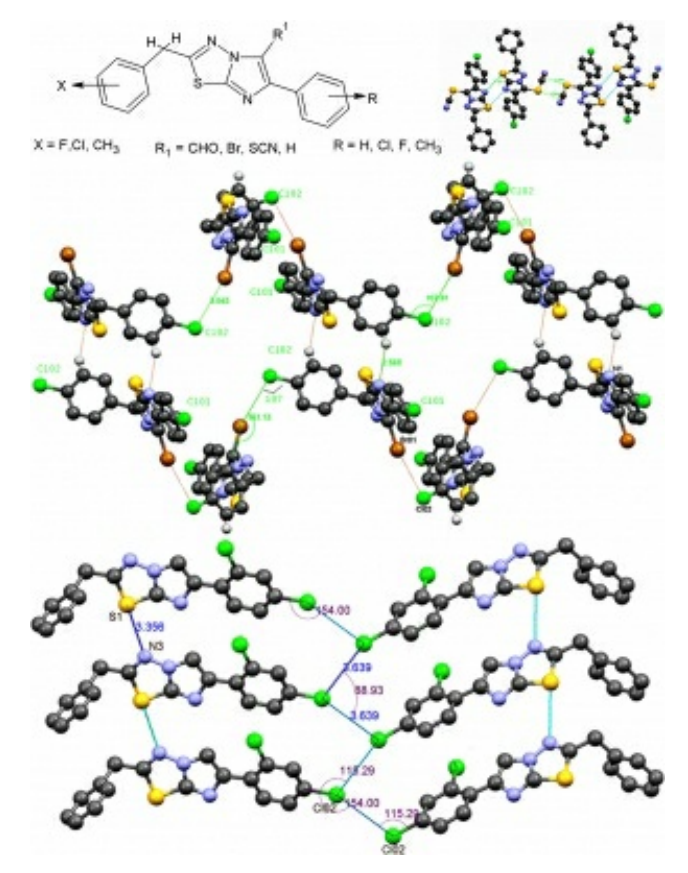

Keywords: Thiadiazoles, Crystal structure, SAR 